\title{
Antireflection $\mathrm{In}_{2} \mathrm{O}_{3}$ Coatings of Self-Organized $\mathrm{TiO}_{2}$ Nanotube Layers Prepared by Atomic Layer Deposition
}

\author{
Jan M. Macak ${ }^{*}, 1$, Jan Prikryl${ }^{1}$, Hanna Sopha ${ }^{1}$ and Lukas Strizik ${ }^{2}$ \\ ${ }^{1}$ Center of Materials and Nanotechnologies, Faculty of Chemical Technology, University of Pardubice, Nam. Cs. Legii 565, 53002 \\ Pardubice, Czech Republic \\ 2 Department of General and Inorganic Chemistry, Faculty of Chemical Technology, University of Pardubice, Studentska 573,53210 \\ Pardubice, Czech Republic
}

Keywords self-organized $\mathrm{TiO}_{2}$ nanotubes, $\mathrm{In}_{2} \mathrm{O}_{3}$, antireflection coating, $\mathrm{ALD}$

*Corresponding author: e-mail jan.macak@upce.cz, Phone: +420 466037401

\begin{abstract}
We report on the uniform anti-reflection coating of $\mathrm{TiO}_{2}$ nanotube layers with a secondary material - indium trioxide $\left(\operatorname{In}_{2} \mathrm{O}_{3}\right)-$ by Atomic Layer Deposition (ALD). We provide for the first time the detailed evidence of the ALD deposited coating inside nanotubes for three different tube layers with aspect ratio up to $\approx 80$, which is so far the highest aspect ratio reported for ALDprocessed self-organized anodic $\mathrm{TiO}_{2}$ nanotubes. We show that uniform $\operatorname{In}_{2} \mathrm{O}_{3}$ coating of the nanotubes strongly influences the overall reflectance of the layers due to intrinsic properties of $\operatorname{In}_{2} \mathrm{O}_{3}$.
\end{abstract}

1 Introduction Self-organized $\mathrm{TiO}_{2}$ nanotubes prepared by electrochemical anodization represent a fascinating material with numerous proven applications $[1,2]$. The potential of $\mathrm{TiO}_{2}$ nanotubes for various functional and advanced devices, in particular when considering all possible tube shapes and geometries that have been achieved until now, has not been extensively exploited. One of the major obstacles to extend the functional range of nanotubes is the difficulty to homogenously coat tube interiors with a secondary material, potentially until all nanotubes are completely full. Starting in 2005, when tube layers with thicknesses over $2 \mu \mathrm{m}$ were first reported [3], researchers carried out efforts to introduce a secondary material into $\mathrm{TiO}_{2}$ nanotubes that resulted in a range of new functionalities of modified layers [4-26]. Until now, several distinct types of deposition techniques that are of reasonable use for the deposition of a secondary material (including metals, quantum dot materials, semiconducting oxides, conducting polymers, dyes and chalcogenides) into self-organized $\mathrm{TiO}_{2}$ nanotubes have been exploited. Electrochemical deposition routes were the first methods investigated for the filing of the tubes by metals [4-7], oxides [8,9] or sulphides [10,11]. Wet chemical routes including in-situ syntheses upon soaking in various precursors of sulphides [12,13], noble metals $[14,15]$ and oxides [16-18], dropping of colloidal solution $[19,20]$ and spin-coating $[21,22]$ represent techniques that have been employed most frequently until now. Recently, also sputtering routes were used to deposit materials inside small aspect ratio $\mathrm{TiO}_{2}$ nanopores [23]. Somewhat surpris- ingly, there are only 3 reports published until now that employed Atomic Layer Deposition (ALD) to provide nanotube layers with a secondary material [24-26]. That is in contrast to numerous reports using ALD to coat porous templates (such as porous alumina [27-29] or polycarbonate [30]) with secondary materials towards inorganic nanostructures. However, aspect ratios of ALD-modified $\mathrm{TiO}_{2}$ nanotubes in these 3 reports [24-26] were rather low, and no direct characterisation or validation (such as thorough SEM inspection) for the presence of deposited materials inside nanotubes, including tube bottoms, was provided. Among various ALD applications, antireflective coatings were produced [31]. However, ALD has not been used in the porous networks of inorganic nanostructures to produce coatings that increase the light trapping within the layers, thus resulting into overall higher antireflection character.

In the present work we report on the uniform antireflection (light trapping) coating of nanotube layers with a secondary material - indium trioxide $\left(\operatorname{In}_{2} \mathrm{O}_{3}\right)$ - by ALD. In particular, we provide detailed evidence of the deposited coating inside nanotubes. We explored two different coating thicknesses and three different tube generations, and their influence on the overall reflectance of the layers. ALD-prepared $\mathrm{In}_{2} \mathrm{O}_{3}$ was found to have lower refractive index and a higher absorption coefficient in the visible and the near-infrared spectral region compared to $\mathrm{TiO}_{2}$. Therefore, we obtain significantly reduced reflectance of the $\mathrm{In}_{2} \mathrm{O}_{3}$ - coated $\mathrm{TiO}_{2}$ nanotubes compared to uncoated ones. 


\section{Results and discussion}

Table 1 gives an overview of the dimensions of all three types of $\mathrm{TiO}_{2}$ nanotube layers employed in this work. Nanotube layers with aspect ratios spanning from 6 to 80 were produced under different experimental conditions using previously published recipes. For all experiments, we performed electrochemical anodization of $\mathrm{Ti}$ substrates $(0.127 \mathrm{~mm}$ thick $)$ in different electrolytes at different times using classical three-electrode configuration and a potentiostat (PGU200V, IPS Elektroniklabor GmbH \& Co. KG). The short tubes were produced in $\mathrm{HF} / \mathrm{H}_{2} \mathrm{SO}_{4}$ electrolytes [1] for 3 hours at $20 \mathrm{~V}$, medium tubes were produced in $\mathrm{NH}_{4} \mathrm{~F}-\mathrm{H}_{2} \mathrm{O}$-glycerol electrolytes [32] for 3 hours at $20 \mathrm{~V}$, and finally long tubes were produced in $\mathrm{NH}_{4} \mathrm{~F}-\mathrm{H}_{2} \mathrm{O}$ ethylene glycol electrolytes [21] for 5 hours at $60 \mathrm{~V}$. All materials were purchased from Sigma-Aldrich. Asprepared nanotubes were submitted to the plasma-assisted $\left(\mathrm{O}_{2}, 50 \mathrm{sccm}\right.$, duration of 100 seconds after each deposition cycle) ALD tool (Ultratech-Cambridge Nanotech Fiji 200) to produce uniform coatings of the tubes consisting of $\mathrm{In}_{2} \mathrm{O}_{3}$. The substrates were heated to $250^{\circ} \mathrm{C}$, pulse duration was 2 seconds. We performed 100 or 300 coating cycles with cyclopentadienylindium (I) (Strem Chemicals, preheated to $75^{\circ} \mathrm{C}$ ) as the precursor, resulting in coatings with a nominal thickness of $\approx 10$ or $\approx 30 \mathrm{~nm}$, respectively, as checked by variable angle spectroscopic ellipsometry (using VASE® ellipsometer, J.A. Woollam, Co., Inc.) on silicon wafers.

Table 1 Dimensions of the tube layers used in this work

\begin{tabular}{llll}
\hline Nanotubes & $\begin{array}{l}\text { Inner tube } \\
\text { diameter }(\mathrm{nm})\end{array}$ & Length $(\mu \mathrm{m})$ & Aspect Ratio* \\
\hline Short & 80 & 0.5 & 6 \\
Medium & 80 & 2 & 25 \\
Long & 100 & 8 & 80 \\
\hline
\end{tabular}

* Aspect ratio $=$ tube length $/$ inner tube diameter

Figure 1 shows scanning electron microscopy (FE-SEM JEOL JSM 7500F) images of $\mathrm{In}_{2} \mathrm{O}_{3}$-coated $\mathrm{TiO}_{2}$ nanotubes layers of all types used in this work. In order to demonstrate the ability of the ALD to coat whole tube interiors including very deep pores of high-aspect ratio nanotubes, we intentionally provide here detailed images on the broken nanotubes at the bottom part of the layer (i.e. at the interface with the underlaying Titanium). For these samples, the ALD tool was set to produce a $10 \mathrm{~nm}$ thin $\mathrm{In}_{2} \mathrm{O}_{3}$ coating, with sufficient diffusion time to enable penetration of the precursor inside the tube pores. The $\mathrm{In}_{2} \mathrm{O}_{3}$ coating was clearly visible inside the nanotubes owing to its different contrast against $\mathrm{TiO}_{2}$. This advantageous contrast has been revealed and exploited based on the different atomic weights, by means of the back-scattering electron detector of the SEM. In the right column of Fig. 1 we show topviews of the identical nanotube layers displayed in the left column. Apparently, the $\operatorname{In}_{2} \mathrm{O}_{3}$ coating nicely surrounded tube openings, thus making them smoother and round compared to their uncoated counterparts (not shown here). In all cases, coatings were present along the whole tube interiors.

Within the ALD tool used, however, considering that the precursors were decomposed using a plasma with a penetration depth limitation (see e.g. Ref. [33] and references therein), we could not obtain uniform coating of nanotubes with aspect ratio larger than 80 . Increasing the number of cycles had no real effect on reaching the deeper pores, owing to the ALD principles [34].

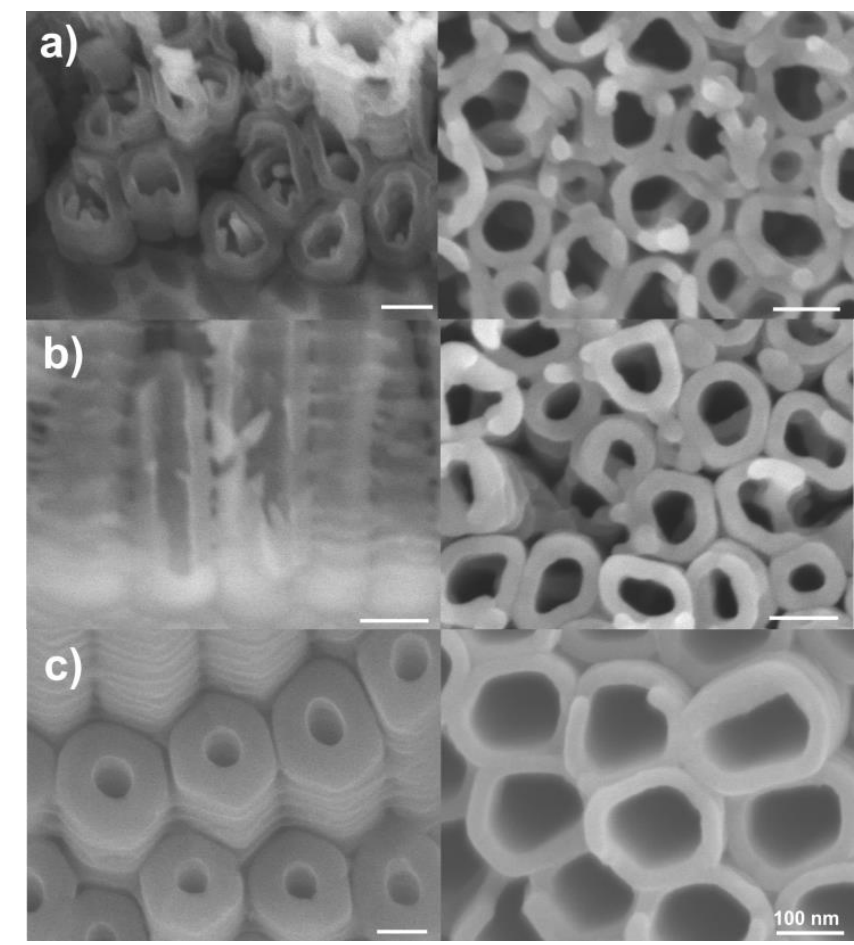

Figure $1 \mathrm{SEM}$ images of (a) short, (b) medium and (c) long $\mathrm{TiO}_{2}$ nanotubes coated with $10 \mathrm{~nm}$ thick $\mathrm{In}_{2} \mathrm{O}_{3}$ layer. Left column shows details on the tubes at the layer bottom part, right column shows top-views. All scale bars represent $100 \mathrm{~nm}$.

Figure 2 shows a detailed view on the $\mathrm{In}_{2} \mathrm{O}_{3}$ coated nanotubes with the aspect ratio of $\approx 80$ at different depth levels. As one can see, the coatings were very uniform, without any visible defects (e.g. cracks) and it follows exactly the tube wall morphology. However, there were apparent differences in the thickness of the coating with a longitudinal increase in the tube length. The coating thickness was reduced from $30 \mathrm{~nm}$ at the uppermost tube part (Fig. 2a) to $20 \mathrm{~nm}$ (Fig. 2b) with a tube depth difference of $2 \mu \mathrm{m}$. The coating thickness further reduced from $20 \mathrm{~nm}$ to $12 \mathrm{~nm}$ with an additional tube depth difference of $2 \mu \mathrm{m}$ (Fig. 2c, in total $4 \mu \mathrm{m}$ depth difference). Finally, the coating thickness further reduced to $5 \mathrm{~nm}$ at the bottom of the nanotubes (Fig. 2d, in total $8 \mu \mathrm{m}$ depth difference). This gradual change in coating thickness represented the 
commonly observed effect within current ALD laboratory tools having limited ability to control the precursor diffusion, when a filling of large aspect ratio nanostructures is carried out.

An additional feature of the ALD process on the nanotube coating can be seen from Fig. 2. Predominantly, the deposited material was observed on the tube interior, however, some very thin coating can be observed also on the tube exteriors, comparably thinner to coated interiors, as marked by an arrow. This exterior coating was observed in particular on the lower aspect ratio nanotube, where small gaps between relatively disordered nanotubes enabled precursor diffusion and subsequent ALD deposition.Thinner exterior coating stems from the fact that tube exteriors have significantly smaller dimensions (gaps with main size of 10-30 nm) in comparison with inner tube diameter (see Table 1). Thus, the precursor would need longer diffusion time than actually used in order to coat these higher aspect ratio gaps, as described in literature [35].

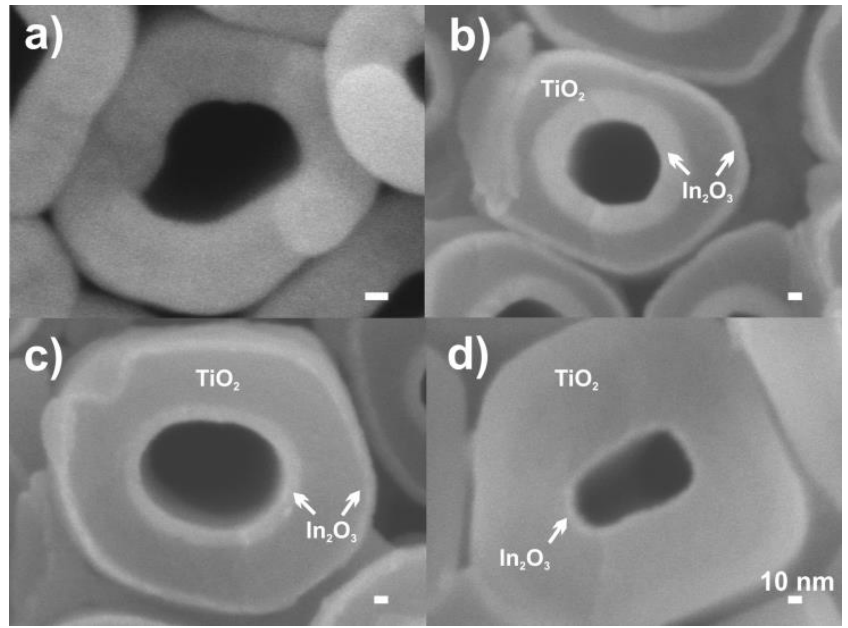

Figure $2 \mathrm{SEM}$ images of $\operatorname{In}_{2} \mathrm{O}_{3}$ - coated $\mathrm{TiO}_{2}$ nanotubes with the aspect ratio of $\approx 80$ at different depth levels: (a) top view with nominal thickness of the coating $\approx 30 \mathrm{~nm}$, tube at the (b) $2 \mu \mathrm{m}$ and (c) $4 \mu \mathrm{m}$ depth from the tube upper surface with thickness of the coating $\approx 20 \mathrm{~nm}$ and $\approx 12 \mathrm{~nm}$, respectively; (d) tube in the depth of $8 \mu \mathrm{m}$ (at the bottom part of the tube layer) with the thickness of the coating $\approx 5 \mathrm{~nm}$. All scale bars represent $10 \mathrm{~nm}$.

This clearly suggests that for nanotubes with a large aspect ratio and smaller gaps, present only within the uppermost part of the layer and absent within the rest of the layer, the exterior coating should not be present in deeper levels of the nanotube layers. However, it is difficult to judge at this stage, whether the tube exterior coatings will influence the overall tube behaviour.

After successful coating of the $\mathrm{TiO}_{2}$ nanotube layers by $\mathrm{In}_{2} \mathrm{O}_{3}$, diffuse reflectance measurements were carried out on all types of nanotubes using UV/VIS/NIR spectrophotometer Jasco V-570 equipped with an integrating sphere (Jasco ISN-470). Figure 3 showed diffuse reflec- tance spectra recorded over a spectral range of 350 up to $800 \mathrm{~nm}$ for short (Fig. 3a), medium (Fig. 3b) and long nanotubes (Fig. 3c). For each tube type, we utilized nanotube layers with $\mathrm{In}_{2} \mathrm{O}_{3}$ coatings of two thicknesses; namely 10 and $30 \mathrm{~nm}$, and for comparison also nanotube layers with no additional ALD-derived coating. As one can see, the reflectance decreases in general within each tube type with increasing $\mathrm{In}_{2} \mathrm{O}_{3}$ coating thickness, even though some minor fluctuations in this trend are apparent. In addition, the reflectance of nanotubes strongly decreases with increasing aspect ratio. The lowest reflectance is obtained for long tubes $(\mathrm{AR} \approx 80)$ with $30 \mathrm{~nm}$ thick $\mathrm{In}_{2} \mathrm{O}_{3}$ coating. Essentially, this means that the more $\mathrm{TiO}_{2}$ and $\mathrm{In}_{2} \mathrm{O}_{3}$ materials present on the illuminated area, the higher is the ability of the layer to absorb light, thus resulting in lower diffuse reflectance. In order to get an idea of how much the reflectance had been decreased, for all types of nanotubes compared to a Ti surface, a reflectance curve for a Ti foil with a native $\mathrm{TiO}_{2}$ layer is shown in Figure 3 (curves marked as „Ti bulk“).

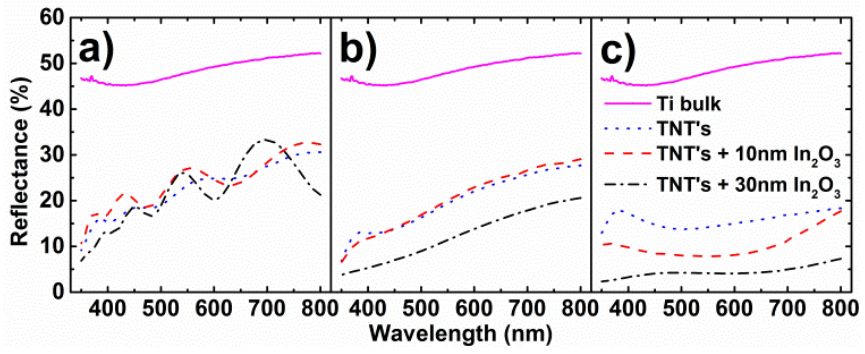

Figure 3 Diffuse reflectance obtained for (a) short, (b) medium and (c) long nanotubes with different thicknesses of $\operatorname{In}_{2} \mathrm{O}_{3}$ coating $(0,10,30 \mathrm{~nm})$. For reference, reflectance of a surface of the Ti foil is provided (marked as "Ti bulk").

In order to analyse the composition of our coatings, XPS analyses were performed using Omicron X-ray source (DAR400, output power $270 \mathrm{~W}$ ) and an electron spectrometer (EA125) attached to a custom built ultra-high vacuum system. Fabricated coatings were analysed by XPS before and after main coating experiments on Si wafers due to a more precise XPS data evaluation compared to nanotube layers. Figure 4a shows XPS spectra recorded on the coated $\mathrm{Si}$ wafer from the same run as nanotubes shown in Fig. 1. Clearly, In $3 \mathrm{~d}$ doublet and $\mathrm{O} 1 \mathrm{~s}$ peaks are revealed. According to the binding energies that match very well with previously published literature [36], the coated layers consist of $\operatorname{In}_{2} \mathrm{O}_{3}$. Somewhat unsurprisingly, the coating displays in particular on its surface, some minor organic contamination and hydroxylation (from-OH groups) that stem from the exposure of samples to the ambient atmosphere rather than from the ALD process itself. However, the level of carbonaceous contamination dramatically decreases in the layer depth according to the XPS depth profiling. There is no significant hydroxylation recorded in depth. 
In order to get a deeper insight into light absorption properties of both oxides, ellipsometric measurements were performed to evaluate optical parameters of the coatings. The same experimental conditions were used as described in Fig. 1. The ellipsometric spectra were measured in the spectral range of 300-2 $300 \mathrm{~nm}$ with a step of $5 \mathrm{~nm}$ and at three angles of light incidence $\left(65^{\circ}, 70^{\circ}\right.$ and $\left.75^{\circ}\right)$. The spectral dependent ellipsometric parameters $\Psi$ and $\Delta$ (where $\Psi$ represents ratio of amplitudes of two perpendicularly polarized waves reflected from the sample surface, and $\Delta$ is their phase difference) were fitted using the optical models: i) $\mathrm{Ti} / \mathrm{In}_{2} \mathrm{O}_{3}$ for $\mathrm{In}_{2} \mathrm{O}_{3}$ coatings and ii) $\mathrm{Ti} / \mathrm{Ti}+\mathrm{TiO}_{2} / \mathrm{TiO}_{2}+$ voids for anodic $\mathrm{TiO}_{2}$ layers.
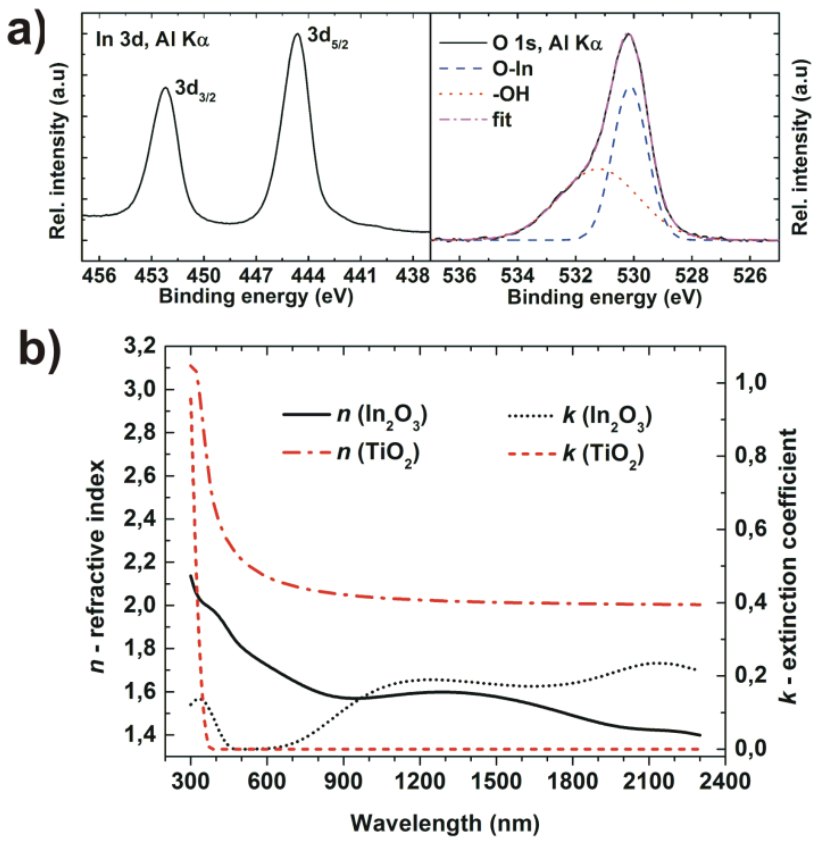

Figure 4 a) XPS spectra of the In $3 d$ doublet peaks and $\mathrm{O} 1 \mathrm{~s}$ peak confirming the $\operatorname{In}_{2} \mathrm{O}_{3}$ composition; b) refractive indexes and extinction coefficients for $\mathrm{In}_{2} \mathrm{O}_{3}$ and $\mathrm{TiO}_{2}$ films

Figure $4 \mathrm{~b}$ shows refractive indexes and extinction coefficients for both $\operatorname{In}_{2} \mathrm{O}_{3}$ (measured on ALD-coated Ti substrates) and $\mathrm{TiO}_{2}$ (measured on anodic oxide on an ultra flat $\mathrm{Ti}$ foil). As one can see from the extinction coefficient of $\mathrm{TiO}_{2}$, having the optical band gap energy $E_{\mathrm{g}} \approx 3.27 \mathrm{eV}$, this oxide does absorb UV light, whereas it is entirely transparent for VIS and IR light. The obtained refractive index is approximately 2.21 at wavelength of $\lambda \approx 500 \mathrm{~nm}$. Both these observations are in complete agreement with existing $\mathrm{TiO}_{2}$ literature on photocatalysis [37]. On the other hand, $\mathrm{In}_{2} \mathrm{O}_{3}$ deposited by ALD does readily absorb not only UV light, but also a significant portion of the VIS light and complete NIR region. Moreover, it has a refractive index of approximately 1.81 at $\lambda \approx 500 \mathrm{~nm}$. These features are essentially responsible for the decreased reflectance of the $\mathrm{In}_{2} \mathrm{O}_{3}$ coated $\mathrm{TiO}_{2}$ nanotubes compared to uncoated counterparts. It has to be noted that the optical con- stants of the $\mathrm{TiO}_{2}$ compact film were obtained using the Tauc-Lorentz parameterization [38], the optical constants of the $\mathrm{In}_{2} \mathrm{O}_{3}$ films were obtained using the combination of the Tauc-Lorentz [38], Gaussian [39] and Lorentz [40] oscillators. Optical constants of Ti substrate with native oxide were known from previous measurements.

\section{Conclusions}

In conclusion, it has been demonstrated that selforganized $\mathrm{TiO}_{2}$ nanotubes with a high aspect ratio $(\approx 80)$ can be uniformly coated with a secondary material consisting of $\operatorname{In}_{2} \mathrm{O}_{3}$, using ALD. The coated layers display considerably stronger light absorption compared to their uncoated counterparts as demonstrated using reflectance measurements. The increased light absorption can be attributed to the instrinsic optical properties of $\operatorname{In}_{2} \mathrm{O}_{3}$, as demonstrated using spectroscopic ellipsometry. The results clearly demonstrate the capability of ALD to coat or completely fill nanotube interiors. Moreover, $\mathrm{TiO}_{2}$ nanotube layers, in particular when coated with $\operatorname{In}_{2} \mathrm{O}_{3}$, showed interesting light trapping capability resulting into strongly increased antireflection character. ALD bears promise for many future applications of nanotube layers.

Acknowledgements ERC is acknowledged for financial support. We would like to thank Prof. Tomas Wagner and Prof. Miloslav Vlcek for their support. Central European Institute of Technology in Brno (CEITEC, Czech Republic) is acknowledged for the ALD access through its Open Access program.

\section{References}

[1] J.M. Macak, H.Tsuchiya, A. Ghicov, K. Yasuda, R. Hahn, S. Bauer, Curr. Opin. Solid StateMater. Sci. 1-2, 3 (2007)

[2] K. Lee, A. Mazare, P. Schmuki, Chem. Rev. 114, 9385 (2014)

[3] J. M. Macak, H. Tsuchiya, P. Schmuki, Angew. Chem. Int. Ed. 44, 2100 (2005)

[4] J.M. Macak, B.G. Gong, M. Hueppe, P. Schmuki, Adv. Mater. 19, 3027 (2007)

[5] J.M. Macak, C. Zollfrank, B.J. Rodriguez, H. Tsuchiya, M. Alexe, P.Greil, P. Schmuki, Adv. Mater. 21, 3121 (2009)

[6] D. Wang, B. Yu, C. Wang, F. Zhou, W. Liu, Adv. Mater. 21, 1964 (2009)

[7] N. Liu, K. Lee, P. Schmuki, Angew. Chem. Int. Ed. 52, $12381(2013)$

[8] L. Assaud, V. Heresanu, M. Hanbücken, L. Santinacci, C.R. Chimie 16, 89 (2013)

[9] Y.Gim, M. Seong, Y.-W. Choi, J. Choi, Electrochem. Commun. 52, 37 (2015)

[10] S. Chen, M. Paulose, Ch. Ruan, G.K. Mor, O.K. Varghese, D. Kouzoudis, C.A. Grimes, J. Photochem. Photobiol. A: Chem 177, 177 (2006)

[11] Y. Yin, Z. Jin, F. Hou, Nanotechnology 18, 495608 (2007)

[12] W.-T.Sun, Y.Yum H-Y. Pan, X.-F. Gao, Q.Chen, L.-M. Peng, J. Am. Chem. Soc. 130, 1124 (2008)

[13] D.R. Baker, P. Kamat, Adv. Func. Mater. 19, 805 (2009)

[14] J.M. Macak, F. Schmidt-Stein, P. Schmuki, Electrochem. Commun. 9, 1783 (2007) 
[15] I. Paramasivam, J.M.Macak, P. Schmuki, Electrochem. Commun. 10, 71 (2008)

[16] A.Benoit, I. Paramasivam, Y.-C. Nah, P. Roy, P. Schmuki, Electrochem. Commun. 11, 728 (2009)

[17] Y.-Y.Son, Z-D.Gao, J.-H. Wang, X.-H. Xia, R. Lynch, Adv. Funct Mater. 21, 1941 (2011)

[18] C.W.Lai, S. Sreekantan, Electrochim.Acta 87, 294 (2013)

[19]J.M. Macak, P. J. Barczuk, H. Tsuchiya, M.Z. Nowakowska, A. Ghicov, M. Chojak, S. Bauer,P.J.Kulesza, P.Schmuki, Electrochem. Commun.7, 1417 (2005)

[20] N.K. Shrestha, J.M. Macak, F. Schmidt-Stein, C.Mierke, B.Fabry, P. Schmuki, Angew. Chem. Int. Ed. 48, 969 (2009)

[21] J. M.Macak, T.Kohoutek, L. Wang, R. Beranek, Nanoscale 5, 9541 (2013)

[22] S.H.Ju, S.Han, J.S.Kim, J.Ind.Eng. Chem. 19, 272 (2013)

[23] J.E. Yoo, K. Lee, M. Altomare, E. Selli, P. Schmuki, Angew. Chem. Int. Ed. 52, 7514 (2013)

[24] S.K. Sarkar et al., J. Phys. Chem. C 14, 8032 (2010)

[25] J. Tupala, M. Kemell, E. Härkönen, M. Ritala and M. Leskelä, Nanotechnology 23, 125707 (2012)

[26] I.Turkevych, S. Kosar, Y. Pihosh, K. Mawatari,T. Kitamori, J. Ye, K. Shimamura, J.Ceram. Soc. Jap. 122, 393 (2014)

[27] M.S. Sander et al, Adv. Mater. 16, 2052 (2004)

[28] M. Kemell et al, Chem. Mater., 19, 1816 (2007)

[29] M. Kemell et al., Nanotechnology 21, 035301 (2010)

[30] H. Sin et al, Adv. Mater. 16, 1197 (2004)

[31] L.G. Zhu et al, IEEE ELECTRON DEVICE LETTERS 33, 1753 (2012)

[32] J.M. Macak, H. Hildebrand, U. Marten-Jahns, P. Schmuki, J. Electroanal. Chem. 621, 254 (2008)

[33] P. Schindler, M. Logar, J. Provine. F.B. Printz, Langmuir 31, 5057 (2015)

[34] S.M. George, Chem. Rev. 110, 111 (2010)

[35] J.M. Elam et al, Chem. Mater. 15, 3507 (2003).

[36] L. Yin et al, J. Mater. Chem. A 2, 18867 (2014)

[37] R. Hofmann, S. T. Martin, W. Choi, D.W. Bahnemann, Chem. Rev. 95, 69 (1995)

[38] G.E. Jellison and F.A. Modine, Appl. Phys. Lett. 69, 371 (1996)

[39] K.-E. Peiponen and E.M. Vartiainen, Phys. Rev. B 44, 8301 (1991)

[40] F. Wooten, Optical Properties of Solids (Academic Press, NY, 1972) p. 52. 